Trabajos Originales

\title{
En mujeres embarazadas con rotura prematura de membranas y edad gestacional entre 34 y 37 semanas: ¿Es la interrupción del embarazo (manejo activo) mejor que el manejo expectante para reducir la sepsis neonatal?
}

\author{
Chuang, Ya-Chuen; González, Conny; Figueroa, Horacio²; Oyarzún, Enrique².
}

1. Internas 6toaño, Medicina, Universidad de los Andes, Santiago de Chile

2. Unidad de Medicina Materno Fetal. Clínica Universidad de los Andes.

\section{RESUMEN}

Introducción: La rotura prematura de membranas (RPM) ocurre en un 8 a 10\% de las embarazadas, y de ellas, un $20 \%$ corresponde a embarazos de pretérmino. El mayor riesgo para el feto luego de una RPM pretérmino son las complicaciones propias de la prematurez. Por debajo de las 34 semanas se favorece el manejo expectante, y el uso de antibióticos y corticoides. Entre las 34 y 37 semanas, sin embargo, las prácticas varían, no habiendo un consenso claro sobre la conducta óptima.

Objetivo: El objetivo de esta revisión es explorar la evidencia actualmente disponible respecto de la conducta activa versus la expectante en embarazos con RPM entre las 34 y 37 semanas (36 semanas más 6 días).

Metodología: Se realizó una búsqueda de literatura médica en distintas bases de datos, dentro de las cuales se incluye "PubMed" y "Cochrane", usando los siguientes términos: "Fetal Membranes, Premature Rupture", "Premature Birth", "34 and 37 weeks" y "Clinical Trial". Se limitó la búsqueda a artículos que fueran ensayos clínicos aleatorizados. De un total de 31 trabajos, se seleccionaron 3, a los cuales se les aplicó la pauta de análisis crítico para evaluación de estudios de terapia.

Resultados: Se incluyeron 3 estudios que respondían a la pregunta planteada. En el primer estudio se concluyó que en pacientes en que hay interrupción inmediata la incidencia de sepsis neonatal es baja y no es posible demostrar que esta conducta mejore los resultados en comparación con el manejo expectante (2.6\% vs. 4.1\%). El manejo activo en este estudio se asoció a mayor incidencia de hiperbilirrubinemia, hipoglicemia, y mayor estadía hospitalaria neonatal. En el segundo artículo se planteó que la incidencia de sepsis neonatal sigue siendo baja, lo cual no disminuyó con la inducción del trabajo de parto. Esta tampoco disminuyó el riesgo de otros resultados neonatales o maternos. Finalmente, el tercer estudio concluyó que la interrupción inmediata aumenta las complicaciones neonatales sin disminución de la sepsis neonatal, pero a expensas de mayor frecuencia de fiebre materna y de hemorragia intraparto.

Conclusiones: El manejo expectante no es inferior al manejo activo en el contexto de RPM entre las semanas 34 a 37 de edad gestacional.

PALABRAS CLAVE: Fetal Membranes, Premature Rupture; Premature Birth; 34 and 37 weeks.

\section{ABSTRACT}

Introduction: Premature rupture of membranes (PROM) occur in eight to ten percent of pregnancies, and 20 percent of them occur in preterm pregnancies. Biggest fetal risks after preterm PROM are complications due to prematurity. Before 34 weeks of gestation it is preferred an expectant management, and the use of antibiotics 
and steroids. Between 34 and 37 weeks, however, practices are variable without a clear consensus about the best management.

Objective: The objective of this review is to explore the available evidence about active versus expectant management in pregnancies with PROM between 34 and 37 weeks (36 weeks plus 6 days).

Methods: Different databases were searched for medical literature, including 'PubMed' and 'Cochrane', using the following terms: 'Fetal Membranes, Premature Rupture', 'Premature Birth', '34 and 37 weeks' and 'Clinical Trial'. The search was limited to clinical randomized trials. From a total of 31 studies, three were selected, in which critical analysis guidelines for evaluation of therapy studies were applied.

Results: Three clinical trials which answered our question were included in this review. The first study concluded that in patients whose pregnancies were interrupted immediately, the incidence of neonatal sepsis was low but is was not able to demonstrate that this action improved outcomes compared to expectant management $(2.6 \%$ vs $4.1 \%)$. Active management in this study was associated to greater incidences of hyperbilirubinemia, hypoglycemia and longer neonatal hospital stay. In the second article the incidence of neonatal sepsis was low and didn't decrease with induction of labor. It also didn't reduce the risk of other maternal nor neonatal outcomes. Finally, the third study concluded that induction of labor increased neonatal complications without reducing neonatal sepsis, but at the expense of increased frequency of intrapartum hemorrhage and maternal fever.

Conclusion: After analyzing the selected articles, it is possible to conclude that there is enough evidence to say that expectant management is not inferior to active management in relation to PROM between 34 and 37 weeks of gestational age.

KEYWORDS: Fetal Membranes; Premature Rupture; Premature Birth; 34 and 37 weeks.

\section{INTRODUCCIÓN}

La rotura prematura de membranas (RPM) es la solución de continuidad espontánea de las membranas ovulares antes del inicio del trabajo de parto. Se denomina de pretérmino cuando ocurre antes de las 37 semanas de gestación, y, en ese contexto, es responsable de un tercio de los partos prematuros. Lo anterior es importante, porque prematurez es la primera causa de mortalidad perinatal y causa importante de morbilidad en la vida adulta.

El manejo de la RPM está influenciado por la edad gestacional (EG) y la presencia de complicaciones como infección clínica, desprendimiento prematuro de placenta, trabajo de parto o estado fetal no tranquilizador.

Desde las 37 semanas de gestación en adelante (término del embarazo), se recomienda el manejo activo para todas las mujeres con RPM, es decir, la interrupción del embarazo. Para lo anterior fue decisivo el trabajo colaborativo canadiense publicado en 1996 que demostró que el manejo activo se asociaba a menor incidencia de morbilidad infecciosa materna y aumento de la satisfacción materna en comparación con el manejo expectante (1). Si bien en embarazos de pretérmino el manejo es expectante (incluyendo el uso de antibióticos y corticoides) para reducir los riesgos perinatales propios de la prematurez, se ha sugerido un manejo diferenciado para los embarazos sobre las 32 semanas.

Específicamente entre las 34 y 37 semanas, las guías nacionales hoy consideran un manejo activo, igual que después de las 37 semanas $(2,3)$. Las razones que consideramos para proponer esa conducta fueron: 1) el pronóstico de los recién nacidos (RN) en ese rango, especialmente mortalidad perinatal (MPN), es similar al de los RN de término, 2) después de las 34 semanas existe en general madurez bioquímica del pulmón fetal, 3) después de las 34 semanas existe relación directa entre el período de latencia e infección, y 4) porque había un trabajo randomizado pequeño que sugería un mejor pronóstico con el manejo activo (4).

Sin embargo, no existe consenso a este respecto. Estados Unidos y Gran Bretaña favorecen la interrupción del embarazo, reconociendo que la evidencia disponible es limitada. Holanda favorece el manejo expectante, y los estudios realizados en Canadá y Australia muestran falta de consenso en el manejo (5-9).

Por otra parte, si bien en la década siguiente al trabajo de Naeff (4) no hubo publicaciones atingentes, en los últimos años esto cambió porque entre 2012 y 2015 se publicaron 3 estudios randomizados que a 
nuestro modo de ver obligan a replantearnos la conducta antes sugerida (10-12).

\section{METODOLOGIA}

Se realizó una búsqueda entre marzo y junio 2017 en las bases de datos Pubmed y Cochrane. En la primera se utilizaron los términos MeSH "Fetal Membranes, Premature Rupture" y "Premature Birth", aplicándose además los filtros de Clinical Trial y publicaciones de los últimos 10 años. Se encontraron 22 artículos, de los cuales 1 respondía a la pregunta establecida. A partir del trabajo encontrado aquí se encontró el segundo trabajo que fue publicado el mismo año como continuación del primero.

En Cochrane se utilizaron los términos "Fetal Membranes, Premature Rupture", "Premature Birth", "34 and 37 weeks" y " Clinical Trial”, encontrándose 9 artículos, de los cuales dos respondían a la pregunta establecida (uno de ellos encontrado anteriormente en PubMed).

La pregunta clínica planteada para realizar la investigación se llevó a cabo según la estrategia PECO (Población expuesta, Exposición, Comparación, Outcome o resultado a evaluar).

Población: Mujeres embarazadas con RPM y EG entre 34 y 37 semanas

Exposición: Manejo activo

Comparación: Manejo expectante

Outcome: Sepsis neonatal

Para la selección de artículos a analizar, los criterios de inclusión fueron: artículos de los últimos 10 años, preferentemente en idioma inglés o español. Sin embargo la búsqueda incluyó otros idiomas y fue realizada por los 3 autores en forma independiente. No se encontraron trabajos en curso. Los criterios de exclusión fueron: edad gestacional bajo 34 semanas o sobre 37 semanas.

\section{RESULTADOS}

A continuación se analizan los estudios seleccionados:

Artículo 1: Induction of labor versus expectant management in women with preterm prelabor rupture of membranes between 34 and 37 weeks: A Randomized controlled trial. David P. van der Ham, Sylvia M. C. Vijgen, Jan G. Nijhuis, et al on behalf of the PPROMEXIL trial group.

PLOS Med 2012. Published online 2012, https://doi.org/10.1371/journal.pmed.1001208

Objetivo
Comprobar si la inducción del trabajo de parto (IoL) en RPM en prematuros de 34-37 semanas reduce la sepsis neonatal sin incrementar la morbilidad del prematuro ni del parto, en comparación con el manejo expectante (EM).

Diseño

Ensayo clínico paralelo, multicéntrico y randomizado en Holanda, entre el 1 de enero 2007 y el 9 de septiembre de 2009, con participación de 60 hospitales. Se incluyeron mujeres cursando embarazos de 34-36+6 semanas, incluyendo gemelares, con diagnóstico de RPM, y pacientes con RPM posterior a las 26 semanas que no habían tenido trabajo de parto y habían alcanzado las 34 semanas. Los criterios de exclusión fueron: embarazo múltiple monocorial, cardiotocografía anormal, líquido amniótico con meconio, signos de infección intrauterina, anomalía fetal mayor, síndrome de HELLP o preeclampsia severa.

Las pacientes que no aceptaron la randomización pero sí autorizaron el uso de su ficha clínica, fueron incluidas en la base de datos y fueron seguidas según sus preferencias.

Las pacientes asignadas al grupo loL, fueron inducidas dentro de las $24 \mathrm{hrs}$ siguientes a la randomización. La inducción fue realizada según las guías clínicas nacionales, con prostaglandinas u ocitocina. En caso de estar indicada operación cesárea, ésta fue realizada lo más pronto posible tras la aleatorización.

Las pacientes asignadas al grupo EM fueron monitorizadas de acuerdo a los protocolos locales hasta el trabajo de parto espontáneo (con al menos un control diario de temperatura y dos hemogramas y PCR semanales). Alcanzadas las 37 semanas de gestación, los partos fueron inducidos según las guías nacionales.

Se registraron resultados maternos y fetales, incluidos el tiempo de sus estadías hospitalarias. La placenta fue enviada para evaluación histológica.

El resultado primario fue sepsis neonatal, definida como: (1) hemocultivo positivo al nacer (excluyendo S. epidermidis) ó (2) dos o más síntomas de infección (apnea, inestabilidad hemodinámica, letargia, intolerancia oral, dificultad respiratoria, inestabilidad termodinámica) 72 horas post parto, más uno de los siguientes: (a) hemocultivo positivo; (b) PCR > $20 \mathrm{mmol} / \mathrm{L}$; (c) cultivo de superficie positivo para patógeno virulento conocido. 
Los resultados secundarios incluyeron morbilidad y mortalidad materna y fetal, y el tiempo de estadía hospitalaria materna y neonatal. Los resultados fueron analizados con intención de tratar, calculados con un intervalo de confianza de $95 \%$, utilizando el SPSS Statistics.

Resultados

536 mujeres dieron el consentimiento informado, 268 fueron randomizadas para el grupo loL y 268 para el grupo EM.

En el grupo de EM, 2 pacientes fueron excluidas para el análisis final y hubo un $14 \%$ de partos por cesárea. En el grupo loL también fueron excluidas 2 pacientes y $13 \%$ resolvió su parto por operación cesárea.

En relación al resultado primario, el grupo loL tuvo 7 casos (2.6\%) de sepsis neonatal y el grupo EM 11 casos $(4.1 \%)$, sin diferencias significativas entre ambos grupos (RR: 0.64, 95\% IC 0.25-1.63; p 0.346).

En cuanto a los resultados neonatales secundarios, el grupo loL presentó mayor incidencia de hipoglicemia (19\% vs. 8.9\%, RR 2.16, 95\% IC 1.363.43; p 0.0008), hiperbilirrubinemia ( $38 \%$ vs. $26 \%$, RR 1.47, 95\% IC 1.13-1.90; p 0.004) y mayor estadía hospitalaria (8 vs. 6.5 días, RR 1.4, 95\% IC 0.11-2.74; p 0.034). El tiempo de estadía en UCI neonatal fue menor en el grupo de manejo activo (4.1 vs. 8.1 días, RR -3.98, 95\% IC -7.89- -0.08; p 0.046).

En relación a los resultados maternos, corioamnionitis clínica ( $2.3 \%$, vs $5.6 \%$, RR $0.4,95 \%$ IC $0.16-1.02$; p 0.045) e histológica (22\% vs $32 \%$, RR $0.69,95 \%$ IC $0.49-0.96$; p 0.026), funisitis (11\% vs $18 \%$, RR $0.61,95 \%$ IC 0.36-1.004; p 0.048) y estadía hospitalaria (9.3 días vs. 11.3 días, RR $-1.94,95 \%$ IC -3.21 -

-0.68; p 0.003) fueron más frecuentes en el grupo EM. Sin embargo sepsis materna fue más frecuente en el grupo loL (6 casos, $2.3 \%$ versus 1 caso, $0.4 \%$ ), aproximándose a la significancia (RR 6, 95\% IC 0.7249.5; p 0.057).

\section{Conclusiones}

No fue posible demostrar ventajas con la interrupción del embarazo en comparación con manejo expectante, en mujeres con RPM entre 34 y 36 +6 semanas de gestación. La interrupción inmediata aumenta las complicaciones neonatales de hipoglicemia, hiperbilirrubinemia y tiempo de estadía hospitalaria, sin reducir sepsis neonatal. Sin embargo, disminuye el riesgo de corioamnionitis materna y funisitis histológica, así como la estadía neonatal en UCl.

Análisis crítico

Este artículo fue publicado en "PLOS Medicine" revista científica con factor de impacto de 13.585 para el año 2015/2016 (13). Dado que se trata de un estudio de tipo "terapia", los autores se plantearon realizar un ensayo clínico randomizado, cuyo análisis del diseño fue realizado mediante las guías CONSORT (Consolidated Standards of Reporting Trials) (14).

El autor principal del estudio está asociado al menos a 15 artículos relacionados con el tema, lo que demuestra que el equipo investigador conocía del tema. El objetivo del trabajo fue bien definido y se describe adecuadamente el método utilizado.

Se calculó el tamaño de la muestra necesaria para demostrar estadísticamente una reducción de riesgo de $66 \%$ con un $80 \%$ de potencia y un $5 \%$ de probabilidad de error, siendo necesario reunir 260 pacientes en cada grupo. Se analizaron 266 pacientes en cada grupo, sin encontrar diferencias en sepsis neonatal. Dado que la diferencia de sepsis entre ambos grupos fue menor de la esperada, el poder resultó insuficiente. No se detalla cómo fueron reclutadas las pacientes, sino sólo quiénes fueron los encargados de realizar el proceso, lo cual podría generar un sesgo de selección.

Las pacientes fueron correctamente aleatorizadas, utilizando un software electrónico, de manera que resultaron dos grupos de características similares. Sin embargo, incluyeron pacientes que tuvieron RPM después de las 26 semanas pero que aún no habían tenido el parto antes de las 34 semanas, factor que podría influir en la incidencia de infección. Esto no porque los grupos no sean comparables (ya que lo son), sino porque no se especifica cuál fue la edad gestacional a la cual ocurrió la rotura prematura de membranas.

Dadas las características de las intervenciones, no se realizó doble ciego, y no se especifica si los que analizaron los resultados eran ciegos al estudio o no. Ningún paciente abandonó el estudio y los resultados fueron analizados por intención de tratar. Todos los resultados fueron expuestos en tablas con valores absolutos y porcentajes, pero no se menciona si hubo 
efectos adversos (solo mencionan que no hubo efectos adversos severos).

Cabe destacar que las conductas clínicas se ejecutaron de acuerdo a protocolos locales, no habiendo por lo tanto un manejo común, lo que genera sesgo de intervención y altera la validez interna del estudio. No puede asegurarse que el resultado primario fuese directa consecuencia de la intervención. Tampoco son extrapolables los datos a cualquier población.

Artículo 2: Management of late preterm premature rupture of membranes: the PPROMEXIL2 trial. Van Der Ham DP, van der Heyden JL, Opmeer BC et al PPROMEXIL-2 trial group

Am J Obstet \& Gynecol 2012. Published online October,

2012 http://dx.doi.org/10.1016/j.ajog.2012.07.024

\section{Objetivo}

El mismo del trabajo anterior. Dada la baja incidencia de sepsis neonatal encontrada en el ensayo clínico PPROMEXIL, y el bajo poder consecuente para constatar diferencias, los autores decidieron realizar un segundo ensayo clínico (PPROMEXIL-2) comparando manejo activo vs. manejo expectante en RPM de 34-37 semanas.

\section{Diseño}

Ensayo clínico multicéntrico randomizado realizado entre Diciembre 2009 y Enero 2011, con posterioridad al estudio PPROMEXIL analizado antes. No hubo cambios en el protocolo ni en la medición de los resultados respecto del estudio anterior (PPROMEXIL).

Se incluyeron 198 mujeres de 60 hospitales de Holanda, cursando embarazos de $34-36+6$ semanas con RPM, y pacientes con RPM posterior a las 26 semanas que no habían tenido el parto a las 34 semanas. Se utilizaron los mismos resultados primarios y secundarios, el mismo protocolo e igual método de análisis.

Se realizó una actualización del metaanálisis realizado por Cochrane Collaboration con respecto a sepsis neonatal, sepsis neonatal con cultivo positivo, SDR y tasa de cesárea incluyendo los datos del ensayo clínico PPROMEXIL trial y el PPROMEXIL-2 trial. Los análisis estadísticos se realizaron en Review Manager Software.

\section{Resultados}

Hubo un total de 198 mujeres randomizadas, 3 de las cuales fueron excluidas porque fueron incluidas con una edad gestacional mayor a $36+6$ semanas. Las 195 mujeres restantes fueron elegibles para el análisis. 100 mujeres fueron asignadas al grupo para inducción del trabajo de parto (loL) y 95 al grupo de manejo expectante (EM). No hubo pérdidas en el seguimiento. Las características de las participantes fueron similares en ambos grupos y la edad gestacional media a la randomización fue 251 días para ambos grupos.

No hubo diferencias significativas en sepsis neonatal entre los dos grupos ( $3 \%$ vs. $4,1 \%$, RR 0.74 , 95\% IC 0.17-3.20; p 0.680). En los resultados secundarios neonatales no hubo diferencias significativas en cuanto a morbilidad y mortalidad. Respecto de los resultados secundarios maternos, en el grupo de manejo expectante fue significativamente mayor la corioamnionitis clínica $(0 \%$ vs. $4.3 \%$, $\mathrm{p}=0.038$ ), la hemorragia $(351 \mathrm{~mL}$ vs. $505 \mathrm{~mL}$, RR -155 , 95\% IC -286--22; p 0.022), y la estadía hospitalaria (8.8 días vs. 13.2 días, RR -4.4, 95\% IC -6.7--2.2; $p$ $<0.001)$.

La actualización del metaanálisis de la base Cochrane, incluyendo los estudios PPROMEXIL y PPROMEXIL-2, no mostró diferencias significativas para ninguno de los resultados analizados, considerando 1428 recién nacidos para sepsis y SDR, y 1417 mujeres para vía de parto.

\section{Conclusiones}

La incidencia de sepsis neonatal sigue siendo baja y no disminuye con la inducción del trabajo de parto. Ésta a su vez no disminuye la ocurrencia de ningún otro resultado materno o neonatal. Los autores concluyeron que este estudio, y la actualización que realizaron del metaánalisis, proveen evidencia a favor del manejo expectante.

Análisis crítico

Este artículo fue publicado en "American Journal of Obstetrics \& Gynecology" revista con factor de impacto 5.574 para el año 2016 (15). El autor principal es el mismo del estudio previo. El protocolo de reclutamiento, así como la forma de aleatorización y el análisis estadístico fueron idénticos a los del estudio original (PPROMEXIL). 
Un aspecto algo confuso es que no se define tamaño muestral necesario. Esto sería prescindible si el presente estudio fuese una extensión del artículo anteriormente expuesto; sin embargo, los autores hacen hincapié en que este es un estudio diferente, por lo que a nuestro parecer debe ser analizado por separado y contener todo lo necesario para darle validez al estudio.

Artículo 3: Immediate delivery compared with expectant management after preterm pre labor rupture of the membranes close to term (PPROMT trial): a randomised controlled trial. J.M. Morris, C.L. Roberts, J.R. Bowen et al on behalf of the PPROMT Collaboration.

The Lancet 2015. Published online November 9, 2015 http://dx.doi.org/10.1016/S0140-6736

(15)00724-2

\section{Objetivo}

Establecer el manejo óptimo luego de rotura prematura de membranas en embarazos de pretérmino tardíos, comparando el manejo activo versus el manejo expectante.

\section{Diseño}

Ensayo clínico randomizado, multicéntrico, conducido en 65 centros de 11 países (Australia, Nueva Zelandia, Argentina, Sudáfrica, Brasil, Reino Unido, Noruega, Egipto, Uruguay, Polonia y Rumania) entre el 28 de Mayo del 2004 y el 30 de Junio del 2013. Se incluyeron mujeres mayores de 16 años con embarazo único, sospecha clínica de RPM, embarazo entre $34+0$ y $36+6$ semanas de edad gestacional y pacientes con RPM < 34 semanas, al cumplir $34+0$ semanas.

Los criterios de exclusión fueron mujeres con trabajo de parto al ingreso, evidencia de corioamnionitis, líquido amniótico meconial o con otras contraindicaciones de continuar el embarazo.

Con posterioridad a la obtención del consentimiento informado, la randomización se realizó en razón de $1: 1$ con programa de randomización computarizado y variables en bloque de 2, 4 y 6 , estratificada por centro.

Para las pacientes asignadas al grupo de interrupción inmediata, ésta se realizó en las primeras 24 horas y la vía de parto fue según indicación obstétrica habitual. En las pacientes asignadas al grupo de manejo expectante se esperó el inicio espontáneo de trabajo de parto o el término (37 semanas) ó interrupción según indicación médica. En estas pacientes se eligió hospitalización versus manejo ambulatorio, uso de antibióticos y seguimiento según las guías locales.

El resultado primario fue la incidencia de sepsis neonatal comprobada o altamente probable. Sepsis neonatal comprobada fue definida como hemocultivo positivo o cultivo de líquido cefalorraquídeo positivo por el cual el recién nacido haya sido tratado por 5 días o más (o haber fallecido antes de los 5 días); o la presencia de uno o más signos clínicos de infección. Sepsis neonatal probable fue definida como la presencia de signos clínicos por los cuales el recién nacido haya sido tratado con antibióticos por 5 días o más, sumado a una o más alteraciones en el laboratorio.

Los resultados secundarios fueron indicador compuesto de mortalidad y morbilidad neonatal severa y morbilidad materna.

Un tamaño muestral de 1812 pacientes era necesario para detectar una reducción a la mitad en la sepsis neonatal $(5 \%$ en el grupo de manejo expectante vs. $2.5 \%$ en el grupo de manejo activo), con un nivel de significancia $5 \%$ de dos colas, y una potencia de $50 \%$. Los resultados fueron analizados con intención de tratar.

\section{Resultados}

1839 pacientes fueron randomizadas; 924 fueron asignadas al grupo de interrupción inmediata, de las cuales 923 fueron incluidas en los análisis (13 de ellas no recibieron la intervención), y hubo 1 pérdida en el seguimiento. 915 fueron asignadas a manejo expectante, de las cuales 912 fueron incluidas en el análisis (1 de ellas no recibió la intervención), y 3 de ellas fueron excluidas ( 2 por retirada y 1 por pérdida de seguimiento).

No hubo diferencias significativas entre ambos grupos. La mediana de edad gestacional, en cada grupo, al momento de la randomización fue de 247 días, y la mediana del tiempo desde la RPM hasta la randomización fue de 30.4 horas en el grupo de manejo activo y 26.4 horas en el grupo de manejo expectante. 
El resultado primario de sepsis neonatal definitiva o probable ocurrió en 23 (2\%) de los 923 neonatos cuyas madres fueron asignadas al grupo de interrupción inmediata, y en 29 (3\%) de los 912 neonatos cuyas madres fueron asignadas a manejo expectante (RR 0.8, 95\% IC 0.5-1.3; p 0.37). No hubo diferencias significativas en el resultado compuesto de mortalidad y morbilidad neonatal; sin embargo, los neonatos nacidos luego de la interrupción inmediata tuvieron menor peso $(\mathrm{p}<0.0001)$, aumento del riesgo de SDR ( $8 \%$ vs. 5\%, RR 1.6, 95\% IC 1.1-2.3; p 0.008), mayor necesidad de ventilación mecánica ( $6 \%$ vs. $4 \%$, RR 1.4, 95\% IC 1.0-1.8; p 0.02), y estadía más prolongada en la UCI neonatal (4 días vs. 2 días, $p<0.0001)$.

En comparación con el grupo de manejo expectante, la interrupción inmediata se asoció con reducción de la hemorragia materna anteparto o intraparto ( $3 \%$ vs. $5 \%$, RR 0.6, 95\% IC 0.4-0.9; p 0.02), menos fiebre intraparto ( $1 \%$ vs. $2 \%$, RR $0.4,95 \%$ IC $0.2-0.9 ;$ p 0.02), menor estadía hospitalaria ( $p<0.0001)$ y mayor número de cesáreas ( $26 \%$ vs. 19\%, RR 1.4, 95\% IC 1.2-1.7; p 0.0001).

\section{Conclusiones}

En mujeres con RPM entre las 34 y $36+6$ semanas de gestación, con feto único y sin contraindicaciones de manejo expectante, la interrupción inmediata aumenta las complicaciones neonatales sin disminución de la sepsis neonatal. Los autores concluyeron que el manejo expectante sería mejor, asumiendo que las pacientes deben vigilarse por el mayor riesgo de hemorragia y fiebre.

\section{Análisis crítico}

Este artículo fue publicado en "The Lancet Medical Journal" revista científica con un factor de impacto de 44.002 para el año 2015 / 2016 (13). El estudio corresponde a un ensayo clínico randomizado, de modo que para el análisis crítico se utilizaron las guías CONSORT (Consolidated Standards of Reporting Trials) (14).

El autor principal del estudio está asociado al menos a 8 artículos relacionados con el tema, lo que le aporta credibilidad a los datos presentados.

El objetivo del trabajo fue bien definido, y describe de forma adecuada el método utilizado para responder esta interrogante. El tamaño de la muestra requerido y cómo se realizó el cálculo fue especificado al igual que el sistema de randomización utilizado. Al contar con el número de pacientes necesario para que la muestra fuera representativa los autores se aseguraron de tener un buen poder estadístico y una alta probabilidad de detectar con significancia estadística el efecto benéfico de una u otra alternativa de manejo.

Los grupos son comparables porque tienen características similares. Si así no fuera se perdería validez interna del estudio.

Un punto importante a considerar es el hecho de que se incluyeron pacientes con RPM antes de las 34 semanas, pero que alcanzaban esa edad gestacional. En este subgrupo, se especificó claramente la edad gestacional a la cual ocurrió la rotura, por lo que el factor tiempo no sería un determinante en contra del estudio, en comparación con los artículos anteriormente analizados. Además la distribución de esas pacientes fue equitativa entre ambos grupos. ( $2 \%$ en manejo activo vs. $4 \%$ en expectante).

Dado que en el manejo de las pacientes se utilizaron guías locales y criterio médico, es posible que haya sesgos de intervención, alterando la validez interna del estudio. Por otra parte, hay que considerar que el tiempo utilizado en reclutar a las pacientes fue de 10 años, período en el que cambió el uso antibiótico, específicamente la amoxicilina con clavulánico, por su relación con enterocolitis necrotizante (16).

El análisis de los resultados se hizo con intención de tratar. Al incluir a aquellas pacientes que después de la randomización no continuaron con el tratamiento indicado, se evita el sesgo de resultado.

\section{DISCUSION}

Rotura prematura de membranas es una patología frecuente en el embarazo, y responsable de un tercio de los partos prematuros en el mundo. Parto prematuro, por su parte, es la causa más importante de morbimortalidad perinatal así como de consecuencias variadas para la vida adulta.

Las guías de manejo de esta patología se modificaron significativamente desde la década de los 90 s, con la demostración de los beneficios de antibióticos y corticoides (antes contraindicados) en los casos de rotura alejada del término de la gestación. 
El manejo, sin embargo, entre las 34 y 37 semanas no es uniforme en los distintos centros perinatales, y si bien nuestra guía nacional sugirió un manejo activo, interrumpiendo el embarazo como si se tratase de embarazos de término, otros centros ofrecen manejo expectante hasta el inicio espontáneo de trabajo de parto. En años recientes se publicaron trabajos clínicos randomizados que obligan a reanalizar la conducta entonces sugerida.

Las publicaciones recientes a las que nos referimos son los tres trabajos que aquí se han discutido. Si bien la RPM cercana al término se asocia con un mayor riesgo de infecciones maternas y perinatales, la interrupción inmediata del embarazo se asocia a un mayor riesgo de patologías asociadas a la prematurez.

Lo anterior es relevante porque en nuestro país ha aumentado la frecuencia de parto prematuro en la última década, y existe preocupación en el mundo por los partos prematuros denominados tardíos, relacionados frecuentemente con inducciones $u$ operaciones cesáreas a destiempo.

Nuestro objetivo fue explorar la evidencia hoy disponible respecto de la conducta activa versus la expectante en embarazos con RPM entre las 34 y 37 semanas (36 semanas más 6 días). Los tres trabajos analizados tienen como resultado primario a analizar la frecuencia de sepsis neonatal, y esto es porque ella es grave, pero además porque si se considerasen otros resultados maternos o perinatales, el tamaño muestral debe aumentar muchísimo para encontrar diferencias significativas.

Debido a las características propias de los estudios, no fue posible realizar doble ciego en ninguno de ellos; sin embargo, consideramos que este factor no representa mayor sesgo para los estudios, ya que hubo criterios claros para los diversos resultados y la ausencia de ciego no afectó la decisión de tratamiento ni el cuidado de la paciente.

Tampoco se hace distinción entre las edades gestacionales a las que se presentaron las distintas patologías, ni está la información detallada respecto de la edad gestacional en que ocurrió la RPM en pacientes bajo las 34 semanas que luego fueron incluidas en el estudio (el último artículo analizado es la excepción a este último punto).
Los dos primeros trabajos analizados no dan cuentan del uso de corticoides, lo que podría influir en la incidencia de patologías respiratorias del neonato.

La incidencia de sepsis neonatal, el resultado primario a evaluar en las tres publicaciones, no fue diferente cualquiera sea el manejo de las pacientes (diferencia no significativa a favor del manejo activo). Por otra parte, la operación cesárea fue significativamente más frecuente en el grupo de manejo activo en el tercer trabajo analizado (el más importante en término de número de pacientes).

El manejo activo (interrupción del embarazo), entre las 34 y $36+6$ semanas, se asoció a mayor incidencia de SDR, ventilación mecánica, hipoglicemia, hiperbilirrubinemia y tiempo de estadía hospitalaria en los recién nacidos. Pero también se asoció a menor incidencia de corioamnionitis, funisitis, fiebre materna, hemorragia y tiempo de estadía en $\mathrm{UCl}$ neonatal.

En relación a los resultados secundarios, a pesar de ser algunos de ellos significativos, se requieren más estudios donde sean considerados como resultados primarios, considerando todas las variables que podrían influir, de modo de alcanzar conclusiones extrapolables a nuestra población.

Cabe destacar que, a pesar de que ambos tipos de manejo tienen sus pros y contras, en los diversos artículos analizados no hacen diferencia en el nivel de gravedad de cada patología. Por ejemplo, no mencionan los niveles de hipoglicemia neonatal o hemorragia materna, siendo tal rango un aspecto relevante ya que puede modificarse el pronóstico y ser diferente el impacto en la vida de la paciente. Por ejemplo la corioamnionitis puede ser un resultado de mayor impacto en comparación con la estadía hospitalaria neonatal en términos de gravedad. Tampoco hubo seguimiento a largo plazo de las madres sometidas a operación cesárea o de los RN diagnosticados con sepsis.

Con un nivel de evidencia $1 \mathrm{~b}$ y un grado de recomendación $A$ (extremadamente recomendable), se puede concluir que el manejo activo no es superior ni inferior al manejo expectante para disminuir la sepsis neonatal (17).

En el número 5 del año 2016 de la Revista Chilena de Obstetricia y Ginecologia, Valdés y Carvajal 
analizaron críticamente el trabajo del Lancet (18) y concluyeron que a partir de esta nueva evidencia, la mejor opción de manejo parecía ser la conducta conservadora. Los autores creen posible, además, replicar este protocolo de modo de reafirmar lo que en él se demuestra pero esta vez en el medio local. Nuestro pensamiento es diferente.

No nos parece necesario realizar nuevos estudios. Creemos que a la luz de la evidencia disponible, y no existiendo diferencias definitivas a favor de una u otra forma de manejo, con ventajas y desventajas para cada una de ellas, los centros perinatales deben adoptar una de ellas y hacer participar a las pacientes de la decisión final. Creemos, además, que una opción razonable y diferente a ofrecer es, para estas pacientes, un manejo como el que ofrecemos entre las 32 y 34 semanas, es decir, hospitalizarlas, administrarle antibióticos y corticoides, e interrumpir el embarazo en 48 horas. Esta conducta nos parece que resulta ser la combinación perfecta en relación a la evidencia hoy disponible.

\section{BIBLIOGRAFÍA}

1) Hannah ME, Ohlsson A, Farine D, et al. Induction of labor compared with expectant management for prelabor rupture of the membranes at term. TERMPROM Study Group. N Engl J Med. 1996; 334(16):1005-10.

2) GUIA PERINATAL_2015_Disponible en: http:// http://web.minsal.cl/wp-

content/uploads/2015/10/GUIAPERINATAL_2015.10.08_web.pdf-R.pdf

3) Enrique Oyarzún Ebensperger y Jose Andres Poblete Lizana. Alto Riesgo Obstétrico. Segunda edición. Ediciones Universidad Catolica de Chile 2013; 201-215 p.

4) Naef RW, Allbert JR, Ross EL, et al. Premature rupture of membranes al 34 to 37 weeks'gestation: aggressive versus conservative management. Am J Obstet Gynecol 1998;178:126-30

5) ACOG Practice Bulletin No.80: premature rupture of membranes. Clinical management for obstetrician-gynecologists. Obstet Gynecol 2007 Apr;109(4):1007-1019 y ACOG practice bulletin no.139: premature rupture of membranes. Obstet Gynecol 2013;122:918-30

6) Royal College of Obstetrics and Gynaecology. Preterm prelabour rupture of membranes (Greentop guideline no.44). 2010. https://www.rcog.org.uk/globalassets/documents/ guidelines/gtg_44pdf.

7) Nederlandse Vereniging voor Obstetric en Gynaecologic. 2002. Rupture of membranes before onset of labor. Disponible en: http://nvogdocumenten.nl/ index.php?pagina=/richtlijn/item/pagina.php\&richtl ijn_id=564.

8) Smith G, Rafuse C, Anand N, et al. Prevalence, management, and outcomes of preterm prelabour rupture of the membranes of women in Canada. $\mathrm{J}$ Obstet Gynaecol Can JOGC. 2005; 27(6):547-53.

9) Buchanan S, Crowther C, Morris J. Preterm prelabour rupture of the membranes: a survey of current practice. Aust N Z J Obstet Gynaecol. 2004;44(5):400-3.

10) van der Ham DP, Vijgen SMC, Nijhuis JG, et al. Induction of labor versus expectant management in women with preterm prelabor rupture of membranes between 34 and 37 weeks: a randomized controlled trial. PLoS Med. 2012; 9(4):e1001208.

11) van der Ham DP, van der Heyden JL, Opmeer BC, et al. Management of late-preterm premature rupture of membranes: the PPROMEXIL-2 trial. Am J Obstet Gynecol. 2012; 207(4):276.e1-10.

12) Morris JM, Roberts CL, Bowen JR, Patterson JA, Bond DM, Algert CS, et al. Immediate delivery compared with expectant management after preterm pre-labour rupture of the membranes close to term (PPROMT trial): a randomised controlled trial. The Lancet. 2016;387(10017):44452.

13) Impact Factor of Plos Medicine - 2016 | 2015 | 2014 | 2013 | 2012 | 2011 | 2010 [Internet]. [citado 1 de julio de 2017]. Disponible en: http://www.scijournal.org/impact-factor-of-PLOSMED.shtml

14) Consort - Welcome to the CONSORT Website [Internet]. [citado 1 de julio de 2017]. Disponible en: http://www.consort-statement.org/

15) American Journal of Obstetrics \& Gynecology [Internet]. [citado 2 de julio de 2017]. Disponible en: http://www.ajog.org/content/impact_factor

16) Kenyon SL, Taylor DJ, Tarnow-Mordi W, ORACLE Collaborative Group. Broad-spectrum antibiotics for spontaneous preterm labour: the ORACLE II randomised trial. ORACLE Collaborative Group. Lancet 2001; 357(9261):989-94. 
17) niveles-grados.pdf [Internet]. [citado 2 de julio de 2017]. Disponible en: http://www.svpd.org/mbe/niveles-grados.pdf

18) Valdes R, Carvajal J Revista de Revistas. Interrupción inmediata vs manejo expectante luego de rotura prematura de membranas cercano al termino del embarazo (ensayo PPROMT): un ensayo controlado randomizado Rev Chil Obstetr Ginecol 2016; 81(5):445-47

\section{TABLA I: RESULTADOS NEONATALES. TABLA RESUMEN DE LOS TRES ARTÍCULOS SELECCIONADOS.}

\begin{tabular}{|c|c|c|c|c|}
\hline & $\begin{array}{l}\text { RESULTADO } \\
\text { NEONATAL }\end{array}$ & $\begin{array}{l}\text { Interrupción Inmediata } \\
{\left[\begin{array}{c}\mathrm{N}=266] \\
N(\%)\end{array}\right.}\end{array}$ & $\begin{array}{c}\text { Manejo Expectante } \\
{\left[\begin{array}{c}{[\mathrm{N}=266]} \\
\mathrm{N}(\%)\end{array}\right.}\end{array}$ & $\operatorname{RR}(p)$ \\
\hline \multirow{6}{*}{ 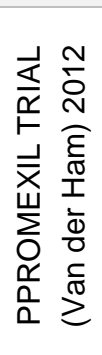 } & $\begin{array}{c}\text { SEPSIS } \\
\text { NEONATAL }\end{array}$ & $7(2,6 \%)$ & $11(4.1 \%)$ & 0.64 (NS) \\
\hline & SDR & $21(7.8 \%)$ & $17(6.3 \%)$ & 1.25 (NS) \\
\hline & HIPOGLICEMIA & 49 (19\%) & $23(8.9 \%)$ & $2.16(0.0008)$ \\
\hline & HIPERBILIRRUB. & $96(38 \%)$ & $67(26 \%)$ & $1.47(0.004)$ \\
\hline & $\begin{array}{c}\text { DS. } \\
\text { HOSPITALIZAC. }\end{array}$ & $8.0[ \pm 7.1 \mathrm{ds}]$. & $6.5[ \pm 7.9$ ds. $]$ & $1.4(0,034)$ \\
\hline & DIAS UCIN & $4.1[ \pm 4.1 \mathrm{ds}]$. & $8.1[ \pm 7.9 \mathrm{ds}]$. & $-3.98(0.046)$ \\
\hline
\end{tabular}

\begin{tabular}{|c|c|c|c|c|}
\hline & $\begin{array}{l}\text { RESULTADO } \\
\text { NEONATAL }\end{array}$ & $\begin{array}{l}\text { Interrupción Inmediata } \\
\qquad \begin{array}{c}{[\mathrm{N}=100]} \\
\mathrm{N}(\%)\end{array}\end{array}$ & $\begin{array}{c}\text { Manejo Expectante } \\
{\left[\begin{array}{c}\mathrm{N}=98] \\
\mathrm{N}(\%)\end{array}\right.}\end{array}$ & $\operatorname{RR}(p)$ \\
\hline \multirow{6}{*}{ 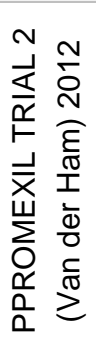 } & $\begin{array}{c}\text { SEPSIS } \\
\text { NEONATAL }\end{array}$ & $3(3 \%)$ & $4(4,1 \%)$ & 0.74 (NS) \\
\hline & SDR & $9(9 \%)$ & $5(5.1 \%)$ & 1.18 (NS) \\
\hline & HIPOGLICEMIA & $8(8.1 \%)$ & $8(8.2 \%)$ & 0.99 (NS) \\
\hline & HIPERBILIRRUB. & $20(20 \%)$ & $21(21 \%)$ & 0.95 (NS) \\
\hline & $\begin{array}{c}\text { DS. } \\
\text { HOSPITALIZAC. }\end{array}$ & $7.4[ \pm 6.1 \mathrm{ds}]$. & 6.9 [ $\pm 6 \mathrm{ds}]$. & 0.52 (NS) \\
\hline & DÍAS UCIN & 7 [7 ds.] & 8 [8.2 ds.] & 0.86 (NS) \\
\hline
\end{tabular}

\begin{tabular}{|c|c|c|c|c|}
\hline & $\begin{array}{l}\text { RESULTADO } \\
\text { NEONATAL }\end{array}$ & $\begin{array}{l}\text { Interrupción Inmediata } \\
{\left[\begin{array}{c}{[\mathrm{N}=923]} \\
\mathrm{N}(\%)\end{array}\right.}\end{array}$ & $\begin{array}{c}\text { Manejo Expectante } \\
{\left[\begin{array}{c}\mathrm{N}=912] \\
\mathrm{N}(\%)\end{array}\right.}\end{array}$ & $\mathbf{R R}(p)$ \\
\hline \multirow{6}{*}{ 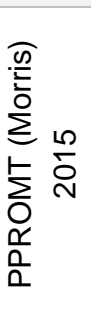 } & SEPSIS NEONATAL & $23(2 \%)$ & $29(3 \%)$ & 0.8 (NS) \\
\hline & $\begin{array}{c}\text { SEPSIS + VM>24 } \\
\text { HRS + MUERTE }\end{array}$ & $79(8 \%)$ & $61(7 \%)$ & $1.2(\mathrm{NS})$ \\
\hline & SDR & $76(8 \%)$ & $47(5 \%)$ & $1.6(0.008)$ \\
\hline & VM (CPAP O ETT) & $114(12 \%)$ & $83(9 \%)$ & $1.4(0.02)$ \\
\hline & DS.HOSPITALIZAC. & 6 [3-10 ds.] & 4 [3-8 ds.] & $(p<0.0001)$ \\
\hline & DÍAS UCIN & 4 [0-10 ds.] & $2[0-7 \mathrm{ds}]$. & $(p<0.0001)$ \\
\hline
\end{tabular}

${ }^{\star} \mathrm{N}$ : número de muestra, RR: riesgo relativo, $p$ : $p$-value, SDR: síndrome de dificultad respiratoria, VM: ventilación mecánica, CPAP: continuous positive airway pressure, ETT: endotracheal tube, DS: días, UCIN: unidad de cuidado intensivo neonatal. NS: no significativo. 
TABLA II: RESULTADOS MATERNOS. TABLA RESUMEN DE LOS TRES ARTíCULOS SELECCIONADOS.

\begin{tabular}{|c|c|c|c|c|}
\hline & $\begin{array}{l}\text { RESULTADO } \\
\text { MATERNO }\end{array}$ & $\begin{array}{l}\text { Interrupción Inmediata } \\
{\left[\begin{array}{c}\mathrm{N}=266] \\
\mathrm{N}(\%)\end{array}\right.}\end{array}$ & $\begin{array}{c}\text { Manejo Expectante } \\
\begin{array}{c}{[\mathrm{N}=266]} \\
\mathrm{N}(\%)\end{array}\end{array}$ & $\mathbf{R R}(p)$ \\
\hline \multirow{7}{*}{ 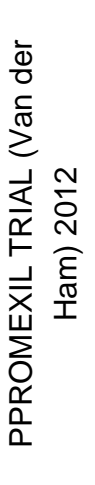 } & $\begin{array}{c}\text { CORIOAMNIONITIS } \\
\text { CLÍNICA }\end{array}$ & $6(2.3 \%)$ & $15(5.6 \%)$ & $0.40(0.045)$ \\
\hline & $\begin{array}{l}\text { CORIOAMNIONITIS } \\
\text { HISTOLÓGICA }\end{array}$ & $43(22 \%)$ & $62(32 \%)$ & $0.69(0.026)$ \\
\hline & $\begin{array}{c}\text { FUNISITIS } \\
\text { HISTOLÓGICA }\end{array}$ & $21(11 \%)$ & $34(18 \%)$ & $0.61(0.048)$ \\
\hline & SEPSIS MATERNA & $6(2.3 \%)$ & $1(0.4 \%)$ & 6.00 (NS) \\
\hline & $\begin{array}{l}\text { HEMORRAGIA } \\
\text { ANTEPARTO }\end{array}$ & $2(0.8 \%)$ & $5(1-9 \%)$ & 0.40 (NS) \\
\hline & CESÁREA & $36(13 \%)$ & 37 (14\%) & 0.98 (NS) \\
\hline & DS. HOSPITALIZAC. & $9.3[ \pm 6.2 \mathrm{ds}]$. & $11.3[ \pm 8.3 \mathrm{ds}]$. & $-1.94(0.003)$ \\
\hline
\end{tabular}

\begin{tabular}{|c|c|c|c|c|}
\hline & $\begin{array}{l}\text { RESULTADO } \\
\text { MATERNO }\end{array}$ & $\begin{array}{l}\text { Interrupción Inmediata } \\
{\left[\begin{array}{c}\mathrm{N}=100] \\
\mathrm{N}(\%)\end{array}\right.}\end{array}$ & $\begin{array}{c}\text { Manejo Expectante } \\
{[\mathrm{N}=98]} \\
\mathrm{N}(\%)\end{array}$ & $\mathbf{R R}(p)$ \\
\hline \multirow{7}{*}{ 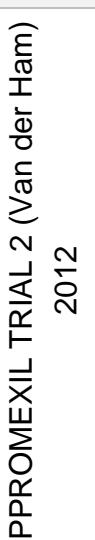 } & $\begin{array}{l}\text { CORIAMNIONITIS } \\
\text { CLINICA }\end{array}$ & $0(0 \%)$ & $4(4.3 \%)$ & $(p 0,038)$ \\
\hline & $\begin{array}{l}\text { CORIOAMNIONITIS } \\
\text { HISTOLÓGICA }\end{array}$ & $12(18 \%)$ & $18(31 \%)$ & 0.64 (NS) \\
\hline & $\begin{array}{c}\text { FUNISITIS } \\
\text { HISTOLÓGICA }\end{array}$ & $6(9.2 \%)$ & $8(31 \%)$ & 0.66 (NS) \\
\hline & SEPSIS MATERNA & $0(0 \%)$ & $0(0 \%)$ & NA \\
\hline & $\begin{array}{l}\text { HEMORRAGIA } \\
\text { ANTEPARTO }\end{array}$ & $1(1 \%)$ & $1(1.1 \%)$ & 0.95 (NS) \\
\hline & CESÁREA & $13(13 \%)$ & $22(22 \%)$ & 0.58 (NS) \\
\hline & DS. HOSPITALIZAC. & 8.8 [ $\pm 5.3 \mathrm{ds}]$. & $13.2[ \pm 9.5 \mathrm{ds}]$. & $-4.4(<0.001)$ \\
\hline
\end{tabular}

**N: número de muestra, RR: riesgo relativo, $p$ : $p$-value , DS: días, NS: no significativo.

\begin{tabular}{|c|c|c|c|c|}
\hline & $\begin{array}{l}\text { RESULTADO } \\
\text { MATERNO }\end{array}$ & $\begin{array}{l}\text { Interrupción Inmediata } \\
{\left[\begin{array}{c}\mathrm{N}=923] \\
\mathrm{N}(\%)\end{array}\right.}\end{array}$ & $\begin{array}{c}\text { Manejo Expectante } \\
\text { [N=912] } \\
\mathrm{N}(\%)\end{array}$ & $\mathbf{R R}(p)$ \\
\hline \multirow{5}{*}{ 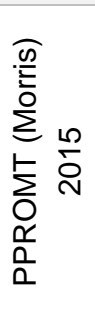 } & $\begin{array}{l}\text { HEMORRAGIA ANTE O } \\
\text { INTRAPARTO }\end{array}$ & $27(3 \%)$ & $46(5 \%)$ & $0.6(0.02)$ \\
\hline & $\begin{array}{l}\text { HEMORRAGIA } \\
\text { POSTPARTO }\end{array}$ & $29(4 \%)$ & $27(3 \%)$ & $1.0(\mathrm{NS})$ \\
\hline & FIEBRE INTRAPARTO & $7(1 \%)$ & $18(2 \%)$ & $0.4(0.02)$ \\
\hline & CESÁREA & $239(26 \%)$ & $196(19 \%)$ & $1.4(0.0001)$ \\
\hline & DS. HOSPITALIZAC. & 5 [3-7 ds.] & 6 [4-9 ds.] & $(p<0.0001)$ \\
\hline
\end{tabular}

${ }^{* \star N} \mathrm{~N}$ número de muestra, RR: riesgo relativo, p: p-value , DS: días, NS: no significativo. 